\title{
An EOQ Model for Varying Items with Weibull Distribution Deterioration and Price-dependent Demand
}

\author{
R. Begum ${ }^{1}$, R. R. Sahoo ${ }^{2}$, S. K. Sahu ${ }^{3}$, and M. Mishra ${ }^{4}$ \\ ${ }^{1}$ Department of Mathematics, Padmanava College of Engineering, Rourkela, Orissa, 769002, India \\ ${ }^{2}$ Department of Physics, Synergy Institute of Technology, Bhubaneswar, Orissa, 752101, India \\ ${ }^{3}$ National Institute of Financial Management, Faridabad, Hariyana, 121001, India \\ ${ }^{4}$ Government Junior Science College, Malkanagiri, Orissa, 768048, India
}

Received 1 July 2009, accepted in final revised form 24 November 2009

\begin{abstract}
In this paper, we have developed an instantaneous replenishment policy for deteriorating items with price-dependent demand. The demand and deterioration rates are continuous and differentiable function of price and time respectively. A variable proportion of the items will deteriorate per time, where shortages are permissible and completely backordered. We have developed a policy with price-dependent demand under profit maximization. The net profit per unit time is a concave function. Further, it is illustrated with the help of a numerical example.
\end{abstract}

Keywords: Price-dependent; Weibull distribution; Varying rate of deterioration.

(c) 2010 JSR Publications. ISSN: 2070-0237 (Print); 2070-0245 (Online). All rights reserved.

DOI: $10.3329 /$ jsr.v2i1.2764

J. Sci. Res. 2 (1), 24-36 (2010)

\section{Introduction}

An optimal replenishment policy is dependent on ordering cost, inventory carrying cost and shortage cost. An important problem confronting a supply manager in any modern organization is the control and maintenance of inventories of deteriorating items. Fortunately, the rate of deterioration is too small for items like steel, hardware, toys, glassware etc. There is little requirement for considering deterioration in the determination of economic lot size. Deterioration is defined as change, damage, decay, spoilage, obsolescence and loss of utility or loss of marginal value of a commodity that results in decreasing usefulness from the original one. The demand rate is assumed to be constant in deterministic inventory models. Covert and Philip [1] considered the assumption of constant deterioration rate to represent the distribution of time to deterioration by using a two-parameter Weibull distribution. Further, Philip [2] generalized this model by assuming a three-parameter Weibull distribution. Misra [3] adopted a two-parameter

\footnotetext{
${ }^{2}$ Corresponding author: rakesh.s24@gmail.com
} 
Weibull distribution deterioration to develop an inventory model with finite rate of replenishment. These investigations were followed by works by several researchers like Shah and Jaiswal [4], Aggarwal [5], Roy-Chaudhury and Chaudhuri [6]. It has been empirically observed that the failure and life expectancy of many items can be expressed in terms of Weibull distribution. This empirical observation has prompted researchers to represent the time of deterioration of a product by a Weibull distribution. The model of Ghare and Schrader [7] was extended by Covert and Philip [1] who obtained an EOQ model with a variable rate of deterioration by assuming a two-parameter Weibull distribution. Later on several other researchers like Tadikamalla [8], Mukhopadhyay et al. [9, 10], Chakrabarty et al. [11] developed economic order quantity models. Therefore, a realistic model is taken in which the deterioration rate is being treated as a time varying function.

A lot of theoretical papers have the assumption that the deterioration rate follows the Weibull distribution. The main attention towards this topic is given by Goyal and Giri [12]. This paper deals with units of the product in stock, which is subjected to deterioration. In this extent the investigations were followed by several researchers like Chakrabarty et al. [11], Chen and Lin [13], Ghosh and Chaudhuri [14], Mahapatra and Maiti [15], Mondal et al. [16], Wu and Lee [17]. Most researchers on the work of deteriorating inventory assumed constant rate of deterioration. However, the Weibull distribution is used to represent the product in stock which deteriorates with time. At first Wagner and Whitin [18] considered an inventory model for goods which deteriorated at the end of a prescribed storage period. Ghare and Schrader [7] revised an economic order quantity model by considering exponential decay in inventory. The further extension to the case of three-parameter Weibull distribution deterioration was done by Shah [19]. Goel and Aggarwal [20] considered a model by assuming varying rate of deterioration.

In real life situation, the retailer's lot size is affected by the demand of the product and the demand is dependent on the price of the product. Therefore, the problems of determining the retail price and lot size are inter-dependent. Kim et al. [21] studied joint price and lot size determination problems for deteriorating products with constant rate of deterioration. Wee [22] also studied the joint pricing and replenishment policy for a deteriorating inventory with price elastic demand rate that decline over time. Abad [23] considered the dynamic pricing and lot sizing problem of perishable goods under partial backlogging of demand. He modeled the backlogging phenomenon using a new approach in which customers are considered impatient. In reality, the effect of marketing policies and the conditions like the price variations and advertisement of an item changes its selling rate among the persons in the recent competitive market. The proper selection to make use of an item, create ideas in customers who are able to make decisions quickly for the selling price of any item. It is commonly seen that lesser selling price causes increase in the selling rate whereas higher selling price has the reverse effect. Hence, the selling rate of an item is dependent on the selling price of that item. This selling rate function must be a decreasing function with respect to the selling price. Several researchers like Ladnay and Sternleib [24], Goyal and Gunasekaran [25], Luo [26], Weng [27], 
Subramanyam and Kumaraswamy [28] and Das et al. [29] developed their models with price variations for deteriorating items. Incorporating this situation, we have developed a policy with price-dependent demand under profit maximization. The failure and life expectancy of many items expressed in terms of Weibull distribution. Therefore we have considered three-parameter Weibull distribution deterioration. Here shortages are permissible and are completely backordered.

\section{Assumptions and Notations}

The following assumptions and notations are considered:

1. The demand rate is dependent on the selling price ' $s$ ' per unit, which is a linear function i.e. $d(s)=a-b s$, where $b>0$ and $s<a=b$.

2. Replenishments are instantaneous with a negligible lead-time.

3. The distribution of the time to deterioration follows a three-parameter Weibull distribution and the deteriorated units are not replaced during a given cycle.

4. The inventory level, replenishment quantity, demand and deterioration are continuous functions of time.

5. The replenishment quantity and period are constant for each cycle.

6. Units are available for satisfying demand immediately after their replenishment.

7. The cost of a deteriorated unit is constant and equal to the unit cost ' $c$ ' .

8. The demand during the stock-out period is completely satisfied by the next replenishment.

9. $\quad T$ is the cycle length, $Q$ is the order quantity per cycle, $T_{1}$ is the deterioration of inventory cycle when there is positive inventory and $\left(T-T_{1}\right)$ is the duration of inventory cycle when stock-out occur.

10. $a, b$ are positive constants. $s$ is the unit selling price, $d(s)$ is the demand rate which is a function of $s$.

11. $c, C_{1}, C_{2}$ and $C_{3}$ is denoted as the unit cost $(c<s)$, fixed production cost per cycle, inventory holding cost, shortage cost per unit backordered per time-unit. All of these cost parameters are positive constant.

12. $I_{1}(t)$ denotes the time-varying inventory level in the cycle segments $t_{1}\left(0 \leq t_{1} \leq T_{1}\right)$. $I_{2}(t)$ is the inventory-level in the cycle segment $t_{2}\left(0 \leq t_{2} \leq\left(T-T_{1}\right)\right)$.

13. $N P\left(T_{1}, s, T\right)$ is the net profit per unit time of inventory system.

14. $T^{*}, T_{1}^{*}, s^{*}, Q^{*}$ and $N P\left(T_{1}^{*}, s^{*}, T\right)$ are the optimal values of the cycle length, inventory, selling price, optimum order rate and net profits respectively.

In this article, the item is assumed to be replenished every $T$ integer time-units. Shortages are completely backordered and satisfied by the next replenishment. The behavior of inventory system is depicted in Fig. 1. It is seen that a review period $T$ is divided into two sub-periods where $T_{1}$ is the period of the system with on-hand inventory and $\left(T-T_{1}\right)$ is the period of the system with shortages. 


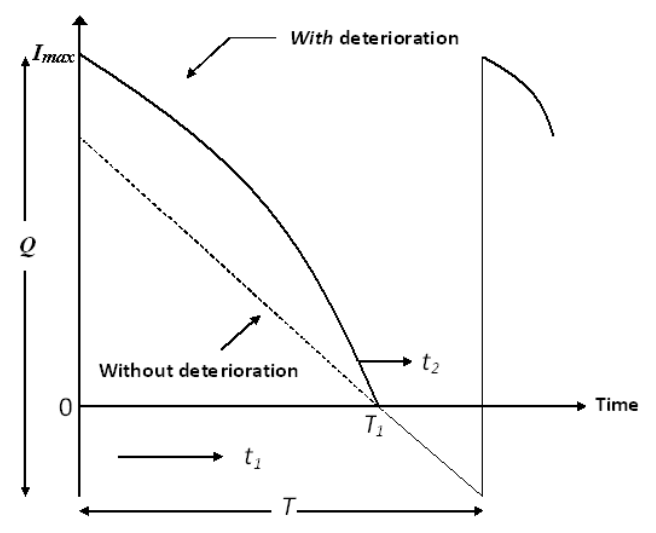

Fig. 1. Inventory system for Weibull distribution deteriorating items.

The rate of deterioration-time relationship for the three-parameter Weibull distribution is shown in Fig. 2. The figure shows that the three-parameter Weibull distribution is most suitable for items with any initial value of the rate of deterioration and for items, which start deteriorating only after a certain period of time.

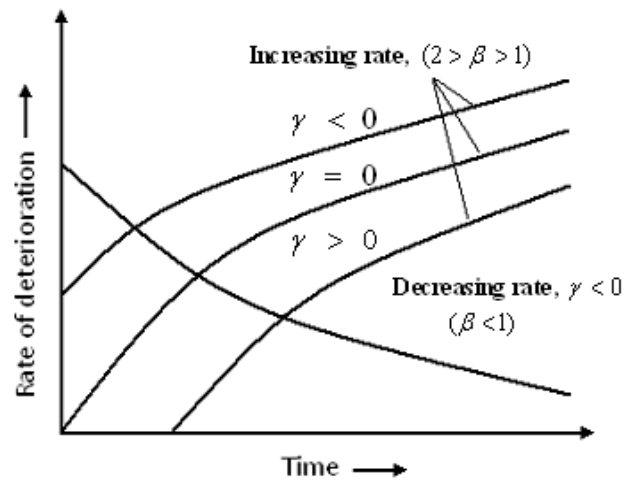

Fig. 2. Rate of deterioration-time relationship for 3-parameter Weibull distribution.

\section{Model Development}

A typical behavior of the inventory system in a cycle is depicted in figure-1. At the beginning of the cycle, the inventory level arrive with its maximum $I_{\max }$ units of item at $t=0$. During the interval $\left[0, T_{1}\right]$, the inventory is depleted due to the combined effects of demand and deterioration. At $T_{1}$, the inventory level is zero and all of the demand during $\left(T-T_{1}\right)$ is backlogged. The total number of backlogged items is replaced by the next replenishment. The distribution of the time to deterioration of the items follows a three- 
parameter Weibull distribution. The three-parameter Weibull density function $f(t)$ from Philip [2] is given by $f(t)=\alpha \beta(t-\gamma)^{\beta-1} e^{-\alpha(t-\gamma)^{\beta}}$

where $f(t)=$ probability density function

$\alpha=$ scale parameter, $\alpha>0$

$\beta$ = shape parameter, $\beta>0$

$t=$ time of deterioration, $t>0$

$\gamma=$ location parameter, $t \geq \gamma$

The instantaneous rate of deterioration of the non-deteriorated inventory at time $t$, $Z(t)$, can be obtained from $Z(t)=\frac{f(t)}{1-F(t)}$, where $F(t)$ is the cumulative distribution function which is equal to $1-e^{-\alpha(t-\gamma)^{\beta}}$ for the three parameter Weibull distribution. Thus, the instantaneous rate of deterioration of the on-hand inventory is $z(t)=\alpha \beta(t-\gamma)^{\beta-1}$. The probability density function represents the deterioration of items which is shown in figure2 , which may have a decreasing, constant or increasing rate of deterioration. It is clear from figure- 2 that the three-parameter Weibull distribution is suitable for items with any initial value of the rate of deterioration and for items, which start deteriorating only after a certain period. The inventory level of the system at time ' $t$ ' over period $[0, T]$ can be described by the following equations:

$$
\frac{d I\left(t_{1}\right)}{d t_{1}}+\theta\left(t_{1}\right) I\left(t_{1}\right)=-d(s), 0 \leq t_{1} \leq T_{1}
$$

with the boundary condition $I(0)=I_{\max }$ and

$$
\frac{d I\left(t_{2}\right)}{d t_{2}}=-d(s), 0 \leq t_{2} \leq\left(T-T_{1}\right)
$$

with the boundary condition $I\left(T_{1}\right)=0$, where $\theta\left(t_{1}\right)=\alpha \beta\left(t_{1}-\gamma\right)^{\beta-1}$ and $d(s)=a-b s$. Using the value of $\theta\left(t_{1}\right)$ in equation (1) and (2), the equations becomes

$$
\frac{d I\left(t_{1}\right)}{d t_{1}}+\alpha \beta\left(t_{1}-\gamma\right)^{\beta-1} I\left(t_{1}\right)=-d(s), 0 \leq t_{1} \leq T_{1}
$$

with the boundary condition $I(0)=I_{\max }$ and

$$
\frac{d I\left(t_{2}\right)}{d t_{2}}=-d(s), 0 \leq t_{2} \leq\left(T-T_{1}\right)
$$

with the boundary condition $I\left(T_{1}\right)=0$. By using the conditions $I(0)=I_{\max }$ and $I\left(T_{1}\right)=0$, the solutions of equation (3) and (4) are respectively given by,

$$
I\left(t_{1}\right)=\frac{I_{\max }-d(s) \int_{0}^{t_{1}} \exp \left[\alpha(t-\gamma)^{\beta}\right] d t}{\exp \left[\alpha(t-\gamma)^{\beta}\right]}, 0 \leq t_{1} \leq T_{1}
$$


where $I_{\max }=d(s) \int_{0}^{T_{1}} \exp \left[\alpha(t-\gamma)^{\beta}\right] d t$ and

$$
I\left(t_{2}\right)=-d(s) t_{2}, 0 \leq t_{2} \leq\left(T-T_{1}\right)
$$

The loss of stock due to deterioration is given by,

$$
D\left(T_{1}\right)=I_{\max }-d(s) T_{1}
$$

Therefore, the total average cost per unit time, $K$ of the system consist of the deterioration cost, the replenishment cost and the backordering cost, which is given by

$$
K=\frac{c D\left(T_{1}\right)}{T}+\frac{C_{1}}{T}+\frac{C_{2}}{T} I_{T}\left(T_{1}\right)+\frac{C_{3}}{T} \int_{0}^{T-T_{1}} d(s) t_{2} d t_{2}
$$

Substituting the values of $D\left(T_{1}\right)$ and $I_{T}\left(T_{1}\right)$ in equation (8), we get

$$
\begin{aligned}
K & =\frac{c d(s) \alpha}{T(1+\beta)}\left[\left(T_{1}-\gamma\right)^{1+\beta}-(-\gamma)^{1+\beta}\right]+\frac{C_{1}}{T}+\frac{C_{2}}{T} d(s)\left[\frac{T_{1}^{2}}{2}+\frac{\alpha T_{1}\left(T_{1}-\gamma\right)^{1+\beta}}{(1+\beta)}\right. \\
& -\frac{\alpha}{(1+\beta)(2+\beta)}\left\{\left(T_{1}-\gamma\right)^{2+\beta}-(-\gamma)^{2+\beta}\right\}-\alpha\left\{\frac{T_{1}\left(T_{1}-\gamma\right)^{1+\beta}-(-\gamma)^{1+\beta} T_{1}}{(1+\beta)}\right\} \\
& \left.+\frac{\alpha \gamma}{(1+\beta)}\left\{\left(T_{1}-\gamma\right)^{1+\beta}-(-\gamma)^{1+\beta}\right\}-\frac{\alpha}{(2+\beta)}\left\{\left(T_{1}-\gamma\right)^{2+\beta}-(-\gamma)^{2+\beta}\right\}\right] \\
& +\frac{C_{3}}{T} d(s) \frac{\left(T-T_{1}\right)^{2}}{2}
\end{aligned}
$$

When there is no shortage, then $T_{1}=T$ and the constant demand reduces to $d(s)=d$.

Putting these values in Eq. (9), we get

$$
\begin{aligned}
& K=\frac{c d \alpha}{T(1+\beta)}\left[\left\{(T-\gamma)^{1+\beta}-(-\gamma)^{1+\beta}\right\}\right]+\frac{C_{1}}{T} \\
& +\frac{C_{2}}{T} d\left[\frac{T^{2}}{2}+\frac{\alpha T(T-\gamma)^{1+\beta}}{(1+\beta)}-\frac{\alpha}{(1+\beta)(2+\beta)}\left\{(T-\gamma)^{2+\beta}-(-\gamma)^{2+\beta}\right\}\right. \\
& -\frac{\alpha}{(2+\beta)}\left\{(T-\gamma)^{2+\beta}-(-\gamma)^{2+\beta}\right\}-\frac{\alpha}{(1+\beta)}\left\{T(T-\gamma)^{1+\beta}-(-\gamma)^{1+\beta} T\right\} \\
& \left.+\frac{\alpha \gamma}{(1+\beta)}\left\{(T-\gamma)^{1+\beta}-(-\gamma)^{1+\beta}\right\}\right]
\end{aligned}
$$

For the constant demand $d(s)=d$ and substituting $\beta=1$, Eq. (9) becomes, 


$$
\begin{aligned}
& K=\frac{c d \alpha}{2 T}\left[\left\{\left(T_{1}-\gamma\right)^{2}-(-\gamma)^{2}\right\}\right]+\frac{C_{1}}{T} \\
& +\frac{C_{2}}{T} d\left[\frac{T_{1}^{2}}{2}+\frac{\alpha T_{1}(T-\gamma)^{2}}{2}-\frac{\alpha}{6}\left\{\left(T_{1}-\gamma\right)^{3}-(-\gamma)^{3}\right\}\right. \\
& \left.-\frac{\alpha}{3}\left\{\left(T_{1}-\gamma\right)^{3}-(-\gamma)^{3}\right\}-\frac{\alpha}{2}\left\{T_{1}\left(T_{1}-\gamma\right)^{2}-(-\gamma)^{2} T_{1}\right\}+\frac{\alpha \gamma}{2}\left\{\left(T_{1}-\gamma\right)^{2}-(-\gamma)^{2}\right\}\right] \\
& +\frac{d C_{3}\left(T-T_{1}\right)^{2}}{2 T}
\end{aligned}
$$

For the condition with no shortage $T_{1}=T$ and substituting $\beta=1$, Eq. (9) becomes,

$$
\begin{aligned}
& K=\frac{c d(s) \alpha}{2 T}\left[\left(T_{1}-\gamma\right)^{2}-(-\gamma)^{2}\right]+\frac{C_{1}}{T}+\frac{C_{2}}{T} d(s)\left[\frac{T_{1}^{2}}{2}+\frac{\alpha T_{1}\left(T_{1}-\gamma\right)^{2}}{2}-\frac{\alpha}{6}\left\{\left(T_{1}-\gamma\right)^{3}-(-\gamma)^{3}\right\}\right. \\
& \left.-\frac{\alpha}{2}\left\{T_{1}\left(T_{1}-\gamma\right)^{2}-(-\gamma)^{2} T_{1}\right\}+\frac{\alpha \gamma}{2}\left\{\left(T_{1}-\gamma\right)^{2}-(-\gamma)^{2}\right\}-\frac{\alpha}{3}\left\{\left(T_{1}-\gamma\right)^{3}-(-\gamma)^{3}\right\}\right]
\end{aligned}
$$

The net profit per unit time, $N P\left(T_{1}, s, T\right)$ is determined by the deduction of the revenue per unit time $(s-c) d(s)$ and the average cost per unit time $K$, which is given by,

Net profit $=$ Revenue - Average cost

$$
N P\left(T_{1}, s, T\right)=(s-c) d(s)-K\left(T_{1}, s, T\right)
$$

As a result,

$$
\begin{aligned}
& N P\left(T_{1}, s, T\right)=(s-c) d(s)-\left[\frac{c \alpha d(s)}{T(1+\beta)}\left\{\left(T_{1}-\gamma\right)^{1+\beta}-(-\gamma)^{1+\beta}\right\}+\frac{C_{1}}{T}+\frac{C_{2}}{T} d(s)\left[\frac{T_{1}^{2}}{2}\right.\right. \\
& +\frac{\alpha T_{1}\left(T_{1}-\gamma\right)^{1+\beta}}{(1+\beta)}-\frac{\alpha}{(1+\beta)(2+\beta)}\left\{\left(T_{1}-\gamma\right)^{2+\beta}-(-\gamma)^{2+\beta}\right\}-\frac{\alpha}{(1+\beta)}\left\{T_{1}\left(T_{1}-\gamma\right)^{1+\beta}-(-\gamma)^{1+\beta} T_{1}\right\} \\
& \left.+\frac{\alpha \gamma}{(1+\beta)}\left\{\left(T_{1}-\gamma\right)^{1+\beta}-(-\gamma)^{1+\beta}\right\}-\frac{\alpha}{(2+\beta)}\left\{\left(T_{1}-\gamma\right)^{2+\beta}-(-\gamma)^{2+\beta}\right\}+\frac{C_{3}}{T} d(s) \frac{\left(T-T_{1}\right)^{2}}{2}\right]
\end{aligned}
$$

For maximizing the net profit $N P\left(T_{1}, s, T\right)$; it can be formulated by the following cases:

Case I: Optimization of $T_{1}$ and $s$ with $T$ given

Our objective is to determine $T_{1}$ and $s$ which maximize the net profit $N P$ with a given $T$. Thus the optimum value of $T_{1}$ and $s$ with $T$ prescribed is the solution of the equation

$$
\frac{\partial N P}{\partial T_{1}}=0 \text { and } \frac{\partial N P}{\partial s}=0
$$


Thus Eq. (15) yields,

$$
\begin{gathered}
\frac{\partial N P}{\partial T_{1}}=-\frac{d(s)}{T}\left[\alpha c\left(T_{1}-\gamma\right)^{\beta}+C_{2}\left\{T_{1}+\alpha T_{1}\left(T_{1}-\gamma\right)^{\beta}-\frac{\alpha\left(T_{1}-\gamma\right)^{\beta+1}}{(1+\beta)}-\alpha\left(T_{1}-\gamma\right)^{\beta}\right.\right. \\
\left.\left.+\alpha \gamma\left(T_{1}-\gamma\right)^{\beta}-\alpha\left(T_{1}-\gamma\right)^{1+\beta}+\frac{\alpha(-\gamma)^{1+\beta}}{(1+\beta)}\right\}-C_{3}\left(T-T_{1}\right)\right]=0
\end{gathered}
$$

and

$$
\begin{aligned}
& \frac{\partial N P}{\partial s}=d(s)+(s-c) d^{\prime}(s)-\frac{d^{\prime}(s)}{T}\left[\frac{c \alpha}{(1+\beta)}\left\{\left(T_{1}-\gamma\right)^{1+\beta}-(-\gamma)^{1+\beta}\right\}+\frac{C_{3}\left(T-T_{1}\right)^{2}}{2}\right. \\
& +C_{2}\left[\frac{T_{1}^{2}}{2}+\frac{\alpha T_{1}\left(T_{1}-\gamma\right)^{1+\beta}}{(1+\beta)}-\frac{\alpha}{(1+\beta)(2+\beta)}\left\{\left(T_{1}-\gamma\right)^{2+\beta}-(-\gamma)^{2+\beta}\right\}\right. \\
& -\frac{\alpha}{(2+\beta)}\left\{\left(T_{1}-\gamma\right)^{2+\beta}-(-\gamma)^{2+\beta}\right\}-\frac{\alpha}{(1+\beta)}\left\{T_{1}\left(T_{1}-\gamma\right)^{1+\beta}-(-\gamma)^{1+\beta} T_{1}\right\} \\
& \left.\left.+\frac{\alpha \gamma}{(1+\beta)}\left\{\left(T_{1}-\gamma\right)^{1+\beta}-(-\gamma)^{1+\beta}\right\}-\frac{\alpha}{(2+\beta)}\left\{\left(T_{1}-\gamma\right)^{2+\beta}-(-\gamma)^{2+\beta}\right\}\right]\right]=0
\end{aligned}
$$

Eqs. (16) and (17) are two simultaneous equations for $T_{1}$ and $s$ provided that they satisfy the sufficient conditions

$$
\frac{\partial^{2} N P}{\partial T_{1}^{2}}<0, \frac{\partial^{2} N P}{\partial s^{2}}<0
$$

and

$$
\left(\frac{\partial^{2} N P}{\partial T_{1}^{2}}\right)\left(\frac{\partial^{2} N P}{\partial s^{2}}\right)-\left(\frac{\partial^{2} N P}{\partial T_{1} \partial s}\right)^{2}>0
$$

The above conditions are verified since for a linear price function of demand $d(s)=a-b s, d^{\prime}(s)=-b$ and $d^{\prime \prime}(s)=0$. For demand, $d(s)>0, T<\infty$; Eq. (16) yields

$$
\begin{aligned}
c \alpha\left(T_{1}-\gamma\right)^{\beta}+ & C_{2}\left\{T_{1}+\alpha T_{1}\left(T_{1}-\gamma\right)^{\beta}-\frac{\alpha\left(T_{1}-\gamma\right)^{\beta+1}}{(1+\beta)}-\alpha\left(T_{1}-\gamma\right)^{\beta}\right. \\
& \left.\left.+\alpha \gamma\left(T_{1}-\gamma\right)^{\beta}-\alpha\left(T_{1}-\gamma\right)^{1+\beta}+\frac{\alpha(-\gamma)^{1+\beta}}{(1+\beta)}\right\}-C_{3}\left(T-T_{1}\right)\right]=0
\end{aligned}
$$

Hence it can be predicted that $\frac{\partial^{2} N P}{\partial T_{1} \partial s}=0$. Therefore, Eqs. (18) and (19) are satisfied for the optimal values of $T_{1}{ }^{*}$ and $s^{*}$. With the optimal values of $T_{1}{ }^{*}$ and $s^{*}$, the net profit can be evaluated from equation (9); and the optimal replenishment lot size is given by

$$
Q^{*}=D\left(T_{1}^{*}\right)+d\left(s^{*}\right) T
$$




\section{Case II: Optimization of $T_{1}$ and $s$ with $T$ as a decision variable}

If $T$ is not prescribed, then for a given $T$; the optimal values of $T_{1}^{*}$ and $s^{*}$, can be found from equation (16) and (17). $N P\left(T_{1}, s, T\right)$ and $Q^{*}$ can be evaluated as in Case-I. Continue the process unless and until get an optimal solution using other values of $T$. The concavity of the net profit is shown in Appendix-A.

\section{Numerical Example}

The parameters are as follows:

Demand rate, $d(s)=25-0.5 s$ units/day,

Item cost $c=\$ 8.00 /$ unit,

Setup cost $c_{1}=\$ 20.00$ /order,

Holding cost $c_{2}=\$ 0.50 /$ unit/day in stock,

Shortage cost $c_{3}=\$ 3.00 /$ unit/day back-ordered, and the items deterioration patterns a

Weibull distribution with $\alpha=0.002,0.05,0.15,0.5,0.1,1.0$ and $\beta=2.0, \gamma=3.0$.

Eqs. (18) and (19) can only be solved numerically with the help of some computer algorithm for a given set of parameter values. For a given $T$, the optimal values of $T_{1}$ and $S$, can be simultaneously found from Eqs. (18) and (19) and the values of $N P\left(T_{1}^{*}, s^{*}, T\right)$ and $Q^{*}$ can be evaluated as in case-I. Repeat the process for other values of $T$ until the best $T$ with its associated $T_{1}$ and $s$ can be found.

Table 1. Optimum solutions obtained using case-II.

\begin{tabular}{llllll}
\hline $\begin{array}{l}\text { Decay } \\
\text { rate } \\
\alpha\end{array}$ & $\begin{array}{l}\text { Cycle length } \\
T \text { (days) }\end{array}$ & $\begin{array}{l}\text { Positive } \\
\text { inventory } \\
T_{1} \text { (days) }\end{array}$ & $\begin{array}{l}\text { Selling price } S \\
(\$)\end{array}$ & $\begin{array}{l}\text { Order rate } \\
Q / T\end{array}$ & $\begin{array}{l}\text { Net profit } \\
N P\end{array}$ \\
\hline & 4 & 3.43004 & 29.4495 & 10.2754 & 14.238 \\
& 5 & 4.27867 & 29.5529 & 10.2264 & 15.3053 \\
0.002 & 6 & 5.11938 & 29.6601 & 10.1807 & 16.7589 \\
& 7 & 5.95271 & 29.7704 & 10.1396 & 18.4427 \\
& 8 & 6.77916 & 29.8835 & 10.1035 & 20.2726 \\
& $9 *$ & $7.59921^{*}$ & $29.9987^{*}$ & $10.0727^{*}$ & $22.1979 *$ \\
\hline & 4 & 3.46119 & 29.9521 & 10.028 & 24.0877 \\
& 5 & 4.15393 & 29.9631 & 10.0698 & 23.2984 \\
& 6 & 4.7570 & 30.0528 & 10.1239 & 24.3342 \\
& 7 & 5.29985 & 30.2006 & 10.1864 & 26.6282 \\
& 8 & 5.79871 & 30.3925 & 10.2515 & 29.8031 \\
\hline 0.15 & $4 *$ & $6.26364 *$ & $30.6188^{*}$ & $10.3144^{*}$ & $33.5972 *$ \\
\hline
\end{tabular}


Table 1 (Continued)

\begin{tabular}{llllll}
\hline & 4 & 1.77438 & 35.3277 & 6.77339 & 97.8422 \\
& 5 & 1.50734 & 34.9295 & 6.69992 & 93.3608 \\
& 6 & 1.29219 & 34.9716 & 6.47452 & 93.0776 \\
& 7 & 1.10717 & 35.2569 & 6.18128 & 95.1028 \\
& 8 & 0.942471 & 35.6894 & 5.85685 & 98.2286 \\
& $9 *$ & $0.792679^{*}$ & $36.2171^{*}$ & $5.51895^{*}$ & $101.694^{*}$ \\
\hline \multirow{4}{*}{0.1} & 4 & -- & 32.0491 & 5.38419 & 59.7334 \\
& 5 & -- & 32.9847 & 4.3572 & 71.8006 \\
& 6 & -- & 33.9614 & 3.60054 & 82.9076 \\
& 7 & -- & 34.9463 & 3.00855 & 92.3704 \\
& 8 & -- & 35.9275 & 2.52781 & 99.9877 \\
& $9 *$ & -- & $36.901 *$ & $2.12762^{*}$ & $105.717 *$ \\
\hline \multirow{4}{*}{1} & 4 & 2.0676 & 40.7091 & 4.33165 & 113.788 \\
& $5 *$ & $1.90381^{*}$ & $39.1307^{*}$ & $4.95741^{*}$ & $114.113^{*}$ \\
& 6 & 1.76527 & 38.3655 & 5.20889 & 112.297 \\
& 7 & 1.64308 & 38.0607 & 5.25943 & 111.035 \\
& 8 & 1.5326 & 38.0408 & 5.19236 & 110.621 \\
& 9 & 1.43102 & 38.2092 & 5.05206 & 110.806 \\
\hline
\end{tabular}

Star $\left(^{*}\right)$ indicates optimal solutions. Hyphen (--) indicates infeasible values.

It is shown from Table 1 that the net profit increases as the deterioration increases whereas the selling price varies slightly. The reason of raise of optimal solution is due to the no shortage constraint in our present model.

\section{Conclusion}

We developed an instantaneous replenishment policy for Weibull deteriorating items with price-dependent demand. Shortages are allowed and completely backordered in the present model. The models with price-dependent demand are surprisingly very few while there is abundance of time-varying demand in inventory models. The fact is that the selling price of an item can affect significantly with the demand of an item. Selling price is the main criterion of the consumer when he/she goes to the market to buy a particular item. The principal feature of the model is the deterministic demand rate which is assumed as a function of selling price. Here, the inventory cycle (or holding time) and selling price optimize the net profit which are simultaneously optimized. Aggarwal and Jaggi [30] developed their model in this approach for decaying inventory. Here a simple heuristic is implemented to derive the best replenishment time interval for a maximum net profit. From the Table-1, it is quite remarkable to note that the selling price of an item slightly changes or remains quite stable as the deterioration rate changes. The threeparameter Weibull distribution deterioration considered here is suitable in optimizing the model as well as control the inventory models. In many realistic situations, stock out is 
unavoidable due to various uncertainties. There are many situations, where the profit of the stored item is higher than its backordered cost. Hence, consideration of shortages is economically desirable in these cases.

In reality, the retailer's lot size is affected by the demand of the product and the demand is dependent on the price of the product. Therefore, the problems of determining the retail price and lot-size are inter-dependent. For reality, the vendor must have some idea about the buyer's behavior such as response to shortages and price. It should be noted that in order to maximize the profit, a vendor can either increase the price or shorten the replenishment cycle or shorten the inventory holding time to counteract a greater loss due to a higher deterioration rate. The selling rate must be a decreasing function with respect to ' $s$ ' as the lower price causes the higher selling rate and vice-versa. In the numerical example, the selling rate is taken as the linear function of ' $s$ '. The model shows how the deterioration patterns influence the scheduling policy and price.

\section{Acknowledgments}

The authors are very much grateful to the referee for his constructive comments for the improvement of the paper.

\section{Appendix A}

$$
\begin{aligned}
& \begin{array}{c}
\frac{\partial N P}{\partial T_{1}}=-\frac{d(s)}{T}\left[\alpha c\left(T_{1}-\gamma\right)^{\beta}+C_{2}\left\{T_{1}+\alpha T_{1}\left(T_{1}-\gamma\right)^{\beta}-\frac{\alpha\left(T_{1}-\gamma\right)^{\beta+1}}{(1+\beta)}-\alpha\left(T_{1}-\gamma\right)^{\beta}\right.\right. \\
\left.\left.+\alpha \gamma\left(T_{1}-\gamma\right)^{\beta}-\alpha\left(T_{1}-\gamma\right)^{1+\beta}+\frac{\alpha(-\gamma)^{1+\beta}}{(1+\beta)}\right\}-C_{3}\left(T-T_{1}\right)\right]=0
\end{array} \\
& \begin{array}{c}
\frac{\partial N P}{\partial s}=d(s)+(s-c) d^{\prime}(s)-\frac{d^{\prime}(s)}{T}\left[\frac{c \alpha}{(1+\beta)}\left\{\left(T_{1}-\gamma\right)^{1+\beta}-(-\gamma)^{1+\beta}\right\}+\frac{C_{3}\left(T-T_{1}\right)^{2}}{2}\right. \\
+C_{2}\left[\frac{T_{1}^{2}}{2}+\frac{\alpha T_{1}\left(T_{1}-\gamma\right)^{1+\beta}}{(1+\beta)}-\frac{\alpha}{(1+\beta)(2+\beta)}\left\{\left(T_{1}-\gamma\right)^{2+\beta}-(-\gamma)^{2+\beta}\right\}\right. \\
-\frac{\alpha}{(2+\beta)}\left\{\left(T_{1}-\gamma\right)^{2+\beta}-(-\gamma)^{2+\beta}\right\}-\frac{\alpha}{(1+\beta)}\left\{T_{1}\left(T_{1}-\gamma\right)^{1+\beta}-(-\gamma)^{1+\beta} T_{1}\right\} \\
\left.+\frac{\alpha \gamma}{(1+\beta)}\left\{\left(T_{1}-\gamma\right)^{1+\beta}-(-\gamma)^{1+\beta}\right\}-\frac{\alpha}{(2+\beta)}\left\{\left(T_{1}-\gamma\right)^{2+\beta}-(-\gamma)^{2+\beta}\right\}\right]=0 \\
+\frac{\partial^{2} N P}{\partial T_{1}^{2}}=-\frac{d(s)}{T}\left[\alpha \beta c\left(T_{1}-\gamma\right)^{\beta-1}+C_{2}\left\{1+\beta T_{1}\left(T_{1}-\gamma\right)^{\beta-1}-\alpha \beta\left(T_{1}-\gamma\right)^{\beta-1}\right.\right. \\
\left.\left.+\alpha \beta\left(T_{1}-\gamma\right)^{\beta-1}-\alpha(1+\beta)\left(T_{1}-\gamma\right)^{\beta}+\right\}+C_{3}\right]
\end{array}
\end{aligned}
$$




$$
\begin{aligned}
& \frac{\partial^{2} N P}{\partial s^{2}}=d^{\prime}(s)+(s-c) d^{\prime \prime}(s)+d^{\prime}(s)-\frac{d^{\prime \prime}(s)}{T}\left[\frac{c \alpha}{(1+\beta)}\left\{\left(T_{1}-\gamma\right)^{1+\beta}-(-\gamma)^{1+\beta}\right\}+\frac{C_{3}\left(T-T_{1}\right)^{2}}{2}\right. \\
& +C_{2}\left[\frac{T_{1}^{2}}{2}+\frac{\alpha T_{1}\left(T_{1}-\gamma\right)^{1+\beta}}{(1+\beta)}-\frac{\alpha}{(1+\beta)(2+\beta)}\left\{\left(T_{1}-\gamma\right)^{2+\beta}-(-\gamma)^{2+\beta}\right\}\right. \\
& -\frac{\alpha}{(2+\beta)}\left\{\left(T_{1}-\gamma\right)^{2+\beta}-(-\gamma)^{2+\beta}\right\}-\frac{\alpha}{(1+\beta)}\left\{T_{1}\left(T_{1}-\gamma\right)^{1+\beta}-(-\gamma)^{1+\beta} T_{1}\right\} \\
& \left.\left.+\frac{\alpha \gamma}{(1+\beta)}\left\{\left(T_{1}-\gamma\right)^{1+\beta}-(-\gamma)^{1+\beta}\right\}\right]\right] \\
& \frac{\partial^{2} N P}{\partial T_{1} \partial s}=\frac{-d^{\prime}(s)}{T}\left[c \alpha\left(T_{1}-\gamma\right)^{\beta}+C_{2}\left\{T_{1}+\alpha T_{1}\left(T_{1}-\gamma\right)^{\beta}-\frac{\alpha\left(T_{1}-\gamma\right)^{\beta+1}}{(1+\beta)}-\alpha\left(T_{1}-\gamma\right)^{\beta}\right.\right. \\
& \left.\left.+\alpha \gamma\left(T_{1}-\gamma\right)^{\beta}-\alpha\left(T_{1}-\gamma\right)^{1+\beta}-\frac{\alpha}{(1+\beta)}\left\{\left(T_{1}-\gamma\right)^{1+\beta}+(1+\beta) T_{1}\left(T_{1}-\gamma\right)^{\beta}-(-\gamma)^{1+\beta}\right\}\right\}-C_{3}\left(T-T_{1}\right)\right]
\end{aligned}
$$

Now the function $\operatorname{NP}\left(T_{1}, s\right)$ will be concave if

$$
\left|\begin{array}{cc}
\frac{\partial^{2} N P}{\partial T_{1}^{2}} & \frac{\partial^{2} N P}{\partial T_{1} \partial s} \\
\frac{\partial^{2} N P}{\partial s \partial T_{1}} & \frac{\partial^{2} N P}{\partial s^{2}}
\end{array}\right|>0
$$

and

$$
\frac{\partial^{2} N P\left(T, s_{1}\right)}{\partial T_{1}^{2}}<0
$$

Using (A3), (A4)and (A5) in (A6); we have

$$
\frac{\partial^{2} N P}{\partial T_{1}^{2}} \frac{\partial^{2} N P}{\partial s^{2}}-\left(\frac{\partial^{2} N P}{\partial T_{1} \partial s}\right)^{2}>0
$$

Also considering the relation (A3), the condition (A7) is verified.

\section{References}

1. R. P. Covert and G.C. Philip, American Institute of Industrial Engineering Transactions 5, 323 (1973).

2. G. C. Philip, AIIE Transactions 6, 159 (1974).

3. R.B. Misra, International Journal of Production Research 13, 495 (1975). doi:10.1080/00207547508943019

4. Y. K. Shah and M. C. Jaiswal, Opsearch 14, 174 (1977).

5. S. P. Aggarwal, Opsearch 15, 184 (1978).

6. M. Roy Chowdhury and K.S. Chaudhuri, Opsearch 20, 99 (1983).

7. P. Ghare and G. Schrader, Journal of Industrial Engineering 14, 238 (1963).

8. P. R. Tadikamalla, AIIE Transactions 10, 108 (1978). 
9. S. Mukhopadhyay, R. N. Mukherjee, and K. S. Chaudhuri, Computers and Industrial Engineering 47, 339 (2004). doi:10.1016/j.cie.2004.06.007

10. S. Mukhopadhyay, R. N.Mukherjee, and K. S. Chaudhuri, International Journal of Mathematical Education in Science and Technology 36, 25 (2005). doi:10.1080/00207390412331303487

11. T. Chakrabarty, B. C. Giri, and K. S. Chaudhuri, Computers \& Operations Research 25, 649 (1998). doi:10.1016/S0305-0548(97)00081-6

12. S. K. Goyal and B. C. Giri, European Journal of Operational Research 134, 1 (2001). doi:10.1016/S0377-2217(00)00248-4

13. J. M. Chen and S. C. Lin, Journal of Information and Optimization Sciences 24, 1 (2003).

14. S. K. Ghosh and K. S. Chaudhuri, Advanced Modelling and Optimization 6, 21 (2004).

15. N. K. Mahapatra and M. Maiti, Computers and Mathematics with Applications 49, 805 (2005). doi:10.1016/j.camwa.2004.07.020

16. B. Mandal, A. K. Bhunia, and M. Maiti, Computers and Industrial Engineering 45, 443 (2003). doi:10.1016/S0360-8352(03)00030-5

17. J. W. Wu and W. C. Lee, Information and Optimization Science 24, 103 (2003).

18. H. M. Wagner and T. M. Whitin, Management Science 5, 89 (1958). doi:10.1287/mnsc.5.1.89

19. Y. Shah, AIIE Transactions 9, 190 (1977).

20. V. P. Goel and S. P. Aggarwal, Proceedings of All India Seminar on OR and Decision Making, University of Delhi, New Delhi, 19 (1981),

21. J. S. Kim, H. Hwang, and S. W. Shinn, Production Planning and Control 6, 45 (1995). doi:10.1080/09537289508930252

22. H. M. Wee, International Journal of Production Economics 40, 163 (1995). doi:10.1016/0925-5273(95)00053-3

23. P. L. Abad, Management Science 42, 1093 (1996). doi:10.1287/mnsc.42.8.1093

24. S. Ladary and A. Sternleib, AIIE Transactions 6, 35 (1974).

25. S. K. Goyal and A. Gunasekaran, Computers and Industrial Engineering 28, 41 (1977).

26. W. Luo, Computers and Industrial Engineering 34, 685 (1998). doi:10.1016/S0360-8352(97)00327-6

27. K. Z. Weng, European Journal of Operational Research 86, 300 (1995). doi:10.1016/0377-2217(94)00104-K

28. S. Subramanyam and S. Kumaraswamy, AIIE Transactions 13, 312 (1981).

29. K. Das, A. K. Bhunia, and M. Maiti, IAPQR Transactions 24, 65 (1999).

30. S. P. Aggarwal and C. K. Jaggi, International Journal of Systems Science 20, 151 (1989). doi:10.1080/00207728908910112 